\title{
Predictors of Preoperative Tinnitus in Unilateral Sporadic Vestibular Schwannoma
}

\author{
Georgios Naros ${ }^{1 *}$, Joey Sandritter ${ }^{1}$, Marina Liebsch ${ }^{1}$, Alex Ofori ${ }^{1}$, Ahmed R. Rizk ${ }^{1}$, \\ Giulia Del Moro ${ }^{1,2}$, Florian Ebner ${ }^{1}$ and Marcos Tatagiba ${ }^{1}$ \\ ${ }^{1}$ Department of Neurosurgery, Eberhard Karls University, Tuebingen, Germany, ${ }^{2}$ Department of Neurosurgery, University of \\ Padova, Padova, Italy
}

Objective: Nearly two-thirds of patients with vestibular schwannoma (VS) are reporting a significantly impaired quality of life due to tinnitus. VS-associated tinnitus is attributed to an anatomical and physiological damage of the hearing nerve by displacing growth of the tumor. In contrast, the current pathophysiological concept of non-VS tinnitus hypothesizes a maladaptive neuroplasticity of the central nervous system to a (hidden) hearing impairment resulting in a subjective misperception. However, it is unclear whether this

OPEN ACCESS

Edited by: Jose Antonio Lopez-Escamez, University of Granada, Spain

Reviewed by:

Angel Batuecas-Caletrio, Complejo Hospitalario de Salamanca, Spain Andrés Soto-Varela,

Complejo Hospitalario Universitario de Santiago,

Spain

*Correspondence: Georgios Naros georgios.naros@ med.uni-tuebingen.de

Specialty section: This article was submitted to Neuro-otology, a section of the journal

Frontiers in Neurology

Received: 02 May 2017 Accepted: 17 July 2017 Published: 03 August 2017

Citation:

Naros G, Sandritter J, Liebsch M, Ofori A, Rizk AR, Del Moro G, Ebner F and Tatagiba M (2017) Predictors of

Preoperative Tinnitus in Unilateral Sporadic Vestibular Schwannoma. Front. Neurol. 8:378. doi: 10.3389/fneur.2017.00378 concept fits to VS-associated tinnitus. This study aims to determine the clinical predictors of VS-associated tinnitus to ascertain the compatibility of both pathophysiological concepts.

Methods: This retrospective study includes a group of 478 neurosurgical patients with unilateral sporadic VS evaluated preoperatively regarding the occurrence of ipsilateral tinnitus depending on different clinical factors, i.e., age, gender, tumor side, tumor size (T1-T4 according to the Hannover classification), and hearing impairment (GardnerRobertson classification, GR1-5), using a binary logistic regression.

Results: $61.8 \%$ of patients complain about a preoperative tinnitus. The binary logistic regression analysis identified male gender [OR 1.90 (1.25-2.75); $p=0.002$ ] and hearing impairment GR3 [OR 1.90 (1.08-3.35); $p=0.026$ ] and GR4 [OR 8.21 (2.29-29.50); $p=0.001$ ] as positive predictors. In contrast, patients with large T4 tumors [OR 0.33 $(0.13-0.86) ; p=0.024]$ and complete hearing loss GR5 [OR $0.36(0.15-0.84) ; p=0.017$ ] were less likely to develop a tinnitus. Yet, $60 \%$ of the patients with good clinical hearing (GR1) and 25\% of patients with complete hearing loss (GR5) suffered from tinnitus.

Conclusion: These data are good accordance with literature about non-VS tinnitus indicating hearing impairment as main risk factor. In contrast, complete hearing loss appears a negative predictor for tinnitus. For the first time, these findings indicate a non-linear relationship between hearing impairment and tinnitus in unilateral sporadic VS. Our results suggest a similar pathophysiology in VS-associated and non-VS tinnitus.

Keywords: tinnitus, vestibular schwannoma, predictors, binary logistic regression, hearing impairment, tumor size

Abbreviations: CI, confidence interval; ENT, ear-nose-throat; GR, Gardner and Robertson; NF, neurofibromatosis; OR, odds ratio; PTA, pure-tone audiometry; VS, vestibular schwannoma. 


\section{INTRODUCTION}

Tinnitus is the second most frequent symptom in vestibular schwannoma (VS) patients (1). Approximately $65-75 \%$ of the VS patients complaint about tinnitus $(2,3)$, in $10 \%$ of the patient it is even the presenting symptom (1). Furthermore, tinnitus is a major factor impairing the VS patients' quality of life (4-6).

The current pathophysiological concept of non-VS tinnitus (e.g., idiopathic tinnitus, tinnitus after auditory or baric trauma) hypothesizes a maladaptive neuroplasticity on a cochlear, brain stem, and/or cortical level as a consequence of (hidden) hearing impairment. These neuroplastic changes are supposed to cause a neuronal hyperexcitability for the residual auditory input resulting in the subjective misperception (7-10). Hence, hearing impairment has been shown to be the strongest predictor for non-VS tinnitus (7-11). In contrast, the pathophysiology of VS-associated tinnitus remains unclear. There are several potential mechanisms of tinnitus generation suggested in the literature (12): (i) ephaptic coupling of cochlear nerve fibers by compression (13), (ii) cochlear dysfunction by ischemia or by biochemical degradation (14), (iii) efferent system dysfunction following compression of the efferent fibers in the inferior vestibular nerve (15), and (iv) cortical reorganization following hearing loss (9). Most studies trying to understand the pathophysiology of VS-associated tinnitus by evaluating its clinical predictors have shown an inverse relationship between tumor size and preoperative tinnitus $(3,12,16-19)$. In contrast to non-VS tinnitus, only few studies have shown an association between preoperative hearing impairment and tinnitus $(3,12,17)$. In a series of 1,000 patients, the rate of tinnitus was higher in hearing than in deaf patients (74 and $46 \%$, respectively) (3). Another study indicates patients with tinnitus to have better hearing than patients without tinnitus, although not reaching statistical significance (17). Similar data are provided by a study in 941 VS patients showing that patients without hearing loss are less likely to experience tinnitus (12). However, no study was able to prove a correlation between tinnitus and hearing impairment, as measured by audiometric thresholds. In contrast, a correlation between patient's age and VS-associated tinnitus was observed $(12,17)$. Finally, several other studies could not establish any predictors of VS-associated tinnitus $(4,5,20)$.

We hypothesize, that in case VS-associated tinnitus is based on similar pathophysiological principles as non-VS tinnitus, a distinct relationship between tinnitus and hearing impairment is expected. Reviewing literature several possible explanations for the inconclusive evidence have been identified. Most studies have applied bivariate statistics that might have obscured the factual relationship between hearing impairment and VS-associated tinnitus. First, there is a known interaction between preoperative hearing impairment and VS size (21). Second, a bivariate analysis assumes a linear correlation; however, the available data imply a more complex relationship between tinnitus, tumor size, and hearing impairment (12). Hence, a multivariate analysis is more appropriate to disentangle clinical predictors of VS-associated tinnitus. However, multivariate statistics necessitates large patient numbers that were available only for few studies $(3,12,17,18)$.

The objective of this study was to determine clinical predictors (i.e., patient age, gender, tumor size, and hearing impairment) of VS-associated tinnitus in a large neurosurgical patient cohort using a multivariate logistic regression.

\section{MATERIALS AND METHODS}

\section{Patients}

All patients enrolled in this retrospective cross-sectional study underwent a neurosurgical removal of a unilateral sporadic VS in the Neurosurgical Department of the University of Tuebingen between January 2008 and January 2015. First, we identified all patients undergoing surgical treatment for intracranial neoplasms of the cranial nerves in this time period $(n=702)$ based on their ICD-10 code (ICD-10: D33.3). 21 patients were excluded due to the histological findings other than VS. Second, we excluded all patients with neurofibromatosis II (ICD-10: Q85.0) and bilateral VS $(n=61)$, patients with relapse or postradiation surgery $(n=40)$ and known contralateral hearing loss [Gardner and Robertson (GR) grading $>2, n=8$ ] resulting in 572 patients for bivariate analysis (Table 1). However, pure-tone audiograms were missing in 94 patients. Hence, only 478 complete datasets were included to the final multivariate statistical analysis (Table 2). Preoperatively, all patients received a clinical evaluation of VS-associated symptoms, a hearing evaluation by an ear-nose-throat specialist (pure-tone audiogram and speech discrimination) and a magnetic resonance imaging of the brain. Patients' characteristics are summarized in Table 1. This study was carried out in accordance with the recommendations of the ethics committee of the Eberhardt Karls University Tuebingen for retrospective studies of data collected as part of routine diagnosis and treatment.

\section{Clinical Evaluation}

All patients underwent a thorough clinical evaluation of VS-associated symptoms (i.e., hearing impairment, tinnitus,

TABLE 1 | Patient cohort.

\begin{tabular}{|c|c|c|c|}
\hline & $\mathrm{TN}-$ & $\mathrm{TN}+$ & \\
\hline Age (years) & $\begin{array}{c}38.5 \%(184 / 478) \\
48.4 \pm 14.1\end{array}$ & $\begin{array}{c}61.5 \%(294 / 478) \\
47.8 \pm 11.7\end{array}$ & $t_{(476)}=0.54 ; p=0.587$ \\
\hline \multicolumn{4}{|l|}{ Gender } \\
\hline $\begin{array}{l}f \\
m\end{array}$ & $\begin{array}{l}63.6 \%(117 / 184) \\
36.4 \%(67 / 184)\end{array}$ & $\begin{array}{l}47.6 \%(140 / 294) \\
52.4 \%(154 / 294)\end{array}$ & $X_{(1)}=11.61 ; p=0.001$ \\
\hline \multicolumn{4}{|l|}{ Tumor side } \\
\hline $\begin{array}{l}\text { Left } \\
\text { Right }\end{array}$ & $\begin{array}{l}45.1 \%(83 / 184) \\
549 \%(101 / 184)\end{array}$ & $\begin{array}{r}466 \%(137 / 294) \\
53.4 \%(157 / 294)\end{array}$ & $X_{(1)}=0.10 ; p=0.750$ \\
\hline \multicolumn{4}{|c|}{ Tumor size (Hannover) } \\
\hline $\begin{array}{l}\text { T1 } \\
\text { T2 } \\
\text { T3 } \\
\text { T4 }\end{array}$ & $\begin{array}{c}3.8 \%(7 / 184) \\
19.6 \%(36 / 184) \\
36.4 \%(67 / 184) \\
40.2 \%(74 / 184)\end{array}$ & $\begin{array}{l}6.8 \%(20 / 294) \\
21.8 \%(64 / 294) \\
44.6 \%(131 / 294) \\
26.9 \%(79 / 294)\end{array}$ & $X_{(3)}=10.18 ; p=0.017$ \\
\hline \multicolumn{4}{|c|}{ Hearing loss (Gardner-Robertson) } \\
\hline $\begin{array}{l}\text { GR1 } \\
\text { GR2 } \\
\text { GR3 } \\
\text { GR4 } \\
\text { GR5 }\end{array}$ & $\begin{array}{c}47.8 \%(88 / 184) \\
22.3 \%(41 / 184) \\
14.7 \%(27 / 184) \\
1.6 \%(3 / 184) \\
13.6 \%(25 / 184)\end{array}$ & $\begin{array}{c}43.2 \%(127 / 294) \\
25.2 \%(74 / 294) \\
20.7 \%(61 / 294) \\
7.8 \%(23 / 294) \\
3.1 \%(9 / 294)\end{array}$ & $X_{(4)}=28.81 ; p<0.001$ \\
\hline
\end{tabular}


TABLE 2 | Logistic regression predicting likelihood of tinnitus based on tumor size, tumor side, hearing impairment, and gender.

\begin{tabular}{|c|c|c|c|c|c|c|c|c|}
\hline & \multirow[t]{2}{*}{$B$} & \multirow[t]{2}{*}{ SE } & \multirow[t]{2}{*}{ Wald } & \multirow[t]{2}{*}{ Df } & \multirow[t]{2}{*}{$p$} & \multirow[t]{2}{*}{$\begin{array}{l}\text { Odds } \\
\text { ratio } \\
\text { (OR) }\end{array}$} & \multicolumn{2}{|c|}{$\begin{array}{c}95 \% \\
\text { Confidence } \\
\text { interval for OR }\end{array}$} \\
\hline & & & & & & & Lower & Upper \\
\hline GR & & & 24.496 & 4 & 0.000 & & & \\
\hline GR2 & 0.345 & 0.252 & 1.877 & 1 & 0.171 & 1.412 & 0.862 & 2.312 \\
\hline GR3 & 0.643 & 0.289 & 4.969 & 1 & 0.026 & 1.903 & 1.081 & 3.350 \\
\hline GR4 & 2.105 & 0.652 & 10.418 & 1 & 0.001 & 8.211 & 2.286 & 29.489 \\
\hline GR5 & -1.026 & 0.431 & 5.663 & 1 & 0.017 & 0.359 & 0.154 & 0.835 \\
\hline SIZE & & & 12.093 & 3 & 0.007 & & & \\
\hline T2 & -0.455 & 0.504 & 0.815 & 1 & 0.367 & 0.634 & 0.236 & 1.704 \\
\hline T3 & -0.370 & 0.483 & 0.586 & 1 & 0.444 & 0.691 & 0.268 & 1.781 \\
\hline $\mathrm{T} 4$ & -1.113 & 0.492 & 5.109 & 1 & 0.024 & 0.329 & 0.125 & .863 \\
\hline AGE & -0.006 & 0.008 & 0.613 & 1 & 0.434 & 0.994 & 0.978 & 1.010 \\
\hline GENDER & 0.618 & 0.202 & 9.359 & 1 & 0.002 & 1.854 & 1.248 & 2.754 \\
\hline SIDE & -0.074 & 0.203 & 0.133 & 1 & 0.715 & 0.929 & 0.624 & 1.382 \\
\hline Constant & 0.947 & 0.605 & 2.448 & 1 & 0.118 & 2.579 & & \\
\hline
\end{tabular}

Tumor size is T2, T3, and T4 compared to T1; hearing impairment is GR2, GR3, GR4, and GR5 compared to T1; and gender is for males compared to females.

dizziness, balance problems, facial palsy, facial dysesthesia, swallowing difficulties, headache, nausea, and vomiting) by a semistructured interview by experienced neurosurgeons. Finally, the presence of ipsilateral tinnitus symptoms was dichotomized for statistical analysis (0: no tinnitus, $\mathrm{TN}-; 1$ : tinnitus present, $\mathrm{TN}+$ ).

\section{Grading of the Hearing Loss}

Hearing impairment was classified according to the GR scale (22). As most of the hearing tests were performed outside of our center, details of the used speech discrimination tests were missing (i.e., word lists, masking, etc.). Hence, grading was based exclusively on the results of the pure-tone audiometry (PTA) resulting in five classes: GR 1 (good, PTA 0-30 dB), GR 2 (serviceable, PTA 31-50 dB), GR 3 (non-serviceable, PTA 51-90 dB), GR 4 (poor, PTA 51-90 dB), and GR 5 (deaf, PTA $0 \mathrm{~dB}$ ). To show the relationship between the absolute hearing impairment and the occurrence of tinnitus, we have reanalyzed the data with narrow binning of the PTA: (1) 0-10 dB, (2) 11-20 dB, (3) 21-30 dB, (4) 31-40 dB, (5) 41-50 dB, (6) 51-60 dB, (7) 61-70 dB, (8) 71-80 dB, (9) 81-90 dB, (10) 91-100 dB, and (11) >100 dB (23).

\section{Tumor Size Classification}

In all patients, a preoperative magnetic resonance image of brain with gadolinium contrast was available, and tumor extent was graded according to Hannover classification (3). VS were classified into four classes: T1 (purely intrameatal), T2 (intra- and extrameatal), T3 (filling the cerebellopontine cistern), and T4 (compressing the brain stem).

\section{Statistics}

All statistical tests were performed using SPSS (IBM SPSS Statistics for Windows, Version 22.0. Armonk, NY, USA: IBM Corp.). Group differences in distribution of clinical attributes such as gender, age, tumor side, tumor size, and preoperative hearing impairment were evaluated by Student's $t$-test or chisquare test. Binary logistic regression analysis was used to determine the predictive value of gender (GENDER), age (AGE), tumor side (SIDE), tumor size (SIZE), and preoperative hearing impairment (GR) for the occurrence of tinnitus (0: TN+ and 1: TN-). Predictive values of the included variable are provided by their odds ratios (OR) together with the $95 \%$ confidence interval (95\% CI). Data are shown as mean \pm standard deviation (SD). Statistical significance was considered with $p<0.05$ for each statistical test.

\section{RESULTS}

The bivariate analysis showed significant differences between $\mathrm{TN}+$ and $\mathrm{TN}-$ patients for gender, tumor size, and hearing impairment. There was a preponderance of male gender, larger tumors (in particular T3), and better hearing function (GR1GR4) in the TM+ group (Figure 1). There were no significant group differences for age and tumor side (Table 1). To ascertain the effects of gender (GENDER), age (AGE), tumor side (SIDE), tumor size (SIZE), and preoperative hearing impairment (GR) on the likelihood of preoperative VS-associated tinnitus, a binomial logistic regression was performed. The logistic regression model was statistically significant $\left[\chi^{2}(10)=52.50, p<0.0001\right]$. Of the five predictor variables GR, SIZE, and GENDER were statistically significant (Table 2 ). The binary logistic regression analysis identified male gender $(p=0.002)$ and hearing impairment GR3 $(p=0.026)$ and GR4 $(p=0.001)$ as positive predictors. Patients with male gender [OR $1.90(1.25-2.75)]$ and higher hearing impairment showed higher odds to exhibit a preoperative tinnitus [GR3: OR 1.90 (1.08-3.35) and GR4: OR 8.21 (2.29-29.50)] than patients with no preoperative hearing impairment (GR1), with severe hearing impairment (GR4) being the strongest predictor of tinnitus. In contrast, large T4 tumors $(p=0.024)$ and complete hearing loss GR5 ( $p=0.017)$ were identified as negative predictors. Patients with large T4 tumors [OR $0.33(0.13-0.86)$ ] and complete hearing loss GR5 [OR $0.36(0.15-0.84)]$ were less likely to develop a tinnitus. However, 43.2\% (127/294) of the patients with good clinical hearing suffer from tinnitus. In contrast, $26.5 \%$ (9/34) of patients with complete hearing loss suffered from tinnitus.

To proof the increased risk of developing tinnitus depending on the hearing impairment, we reanalyzed the data with narrow binning of the PTA values. A binomial logistic regression was performed to show the predictive value of the PTA for the presence of tinnitus. The logistic regression model was statistically significant $\left[\chi^{2}(10)=32.17, p<0.0001\right]$ with PTA being a significant predictor of tinnitus $(p=0.002)$. Patients with PTA $>100 \mathrm{~dB}$ were less likely to present a preoperative tinnitus than other patients (Table 3). However, for patients with residual hearing, there was positive relationship between hearing impairment and the risk of tinnitus occurrence (Figure 2).

\section{DISCUSSION}

This study evaluated the clinical predictors of preoperative tinnitus in unilateral sporadic VS patients indicating male gender and 

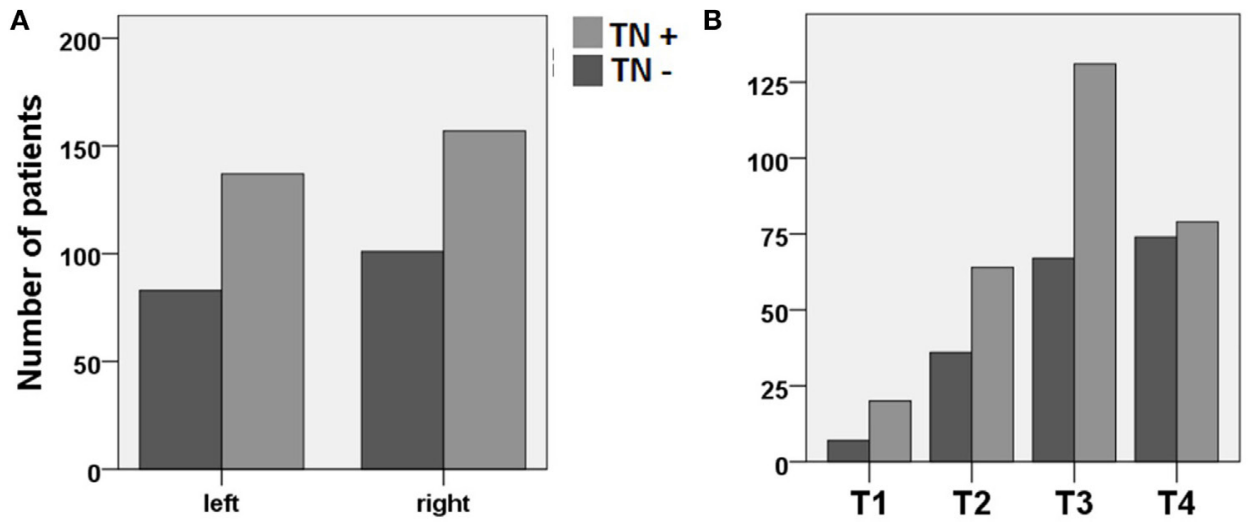

$\mathrm{TN}+$

TN -
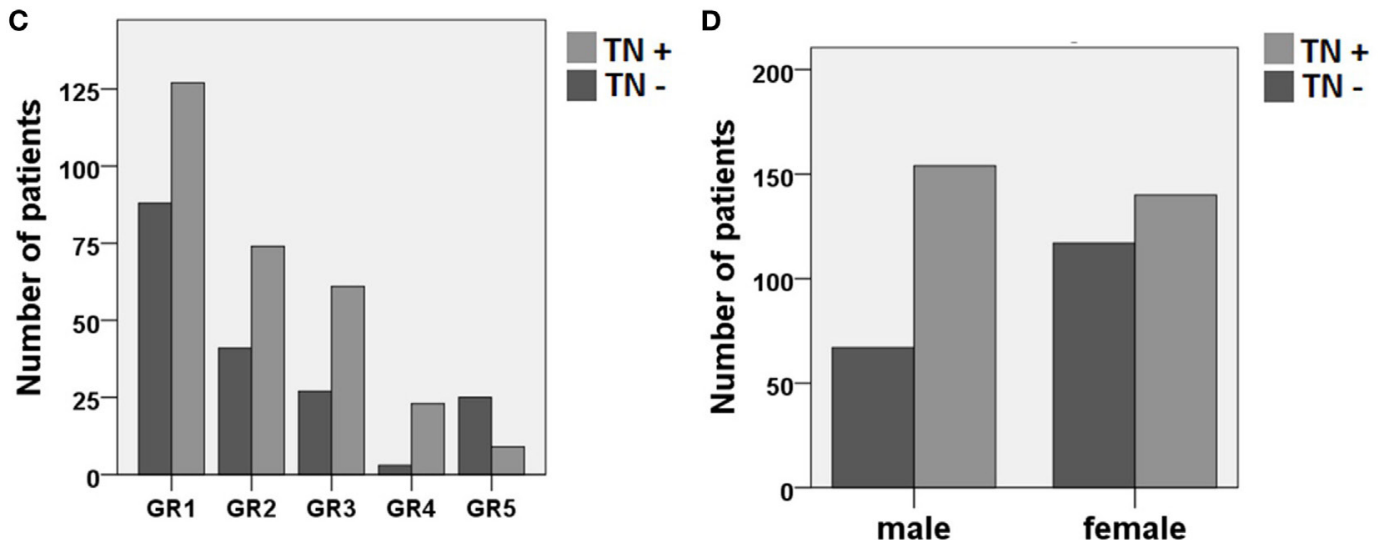

FIGURE 1 | Distribution of patients with (TN+) and without (TN-) preoperative vestibular schwannoma-associated tinnitus depending on (A) tumor side, (B) tumor size (Hannover classification, T1-T4), (C) preoperative hearing impairment (Gardner and Robertson scale, GR1-GR5), and (D) gender.

TABLE 3 | Logistic regression predicting likelihood of tinnitus based on pure-tone audiometry (PTA).

\begin{tabular}{llllc}
$B \quad$ SE Wald Df $p$ & $\begin{array}{c}\text { Odds } \\
\text { ratio } \\
\text { (OR) }\end{array}$ & $\begin{array}{c}95 \% \\
\text { Confidence } \\
\text { interval for OR }\end{array}$ \\
\hline & & Lower Upper
\end{tabular}

\begin{tabular}{|c|c|c|c|c|c|c|c|c|}
\hline PTA & & & 27.438 & 10 & 0.002 & & & \\
\hline $0-10 \mathrm{~dB}$ & 1.452 & 0.527 & 7.587 & 1 & 0.006 & 4.274 & 1.520 & 12.012 \\
\hline & 1.419 & 0.440 & & 1 & & & & \\
\hline & 1.233 & 0.452 & & 1 & & & & 8.322 \\
\hline & 2.039 & 0.481 & & 1 & & & & 9.708 \\
\hline & & 0.4 & & 1 & & & & \\
\hline & & & & 1 & & & & \\
\hline & & & & 1 & & & & \\
\hline & 1.71 & 0.6 & & 1 & & & & 20.722 \\
\hline $81-90$ & 2.200 & 0.691 & .128 & 1 & & 9.0 & & 35.003 \\
\hline $91-100 \mathrm{~dB}$ & 2.771 & 0.667 & 17.269 & 1 & 0.000 & 15.972 & 4.323 & 59.010 \\
\hline Constant & -1.022 & 0.389 & 6.907 & 1 & 0.009 & 0.360 & & \\
\hline
\end{tabular}

ORs are provided compared to PTA $>100 \mathrm{~dB}$.

hearing impairment as positive predictors of tinnitus. In contrast, large tumors and complete hearing loss were disentangled as negative predictors in our patient cohort.

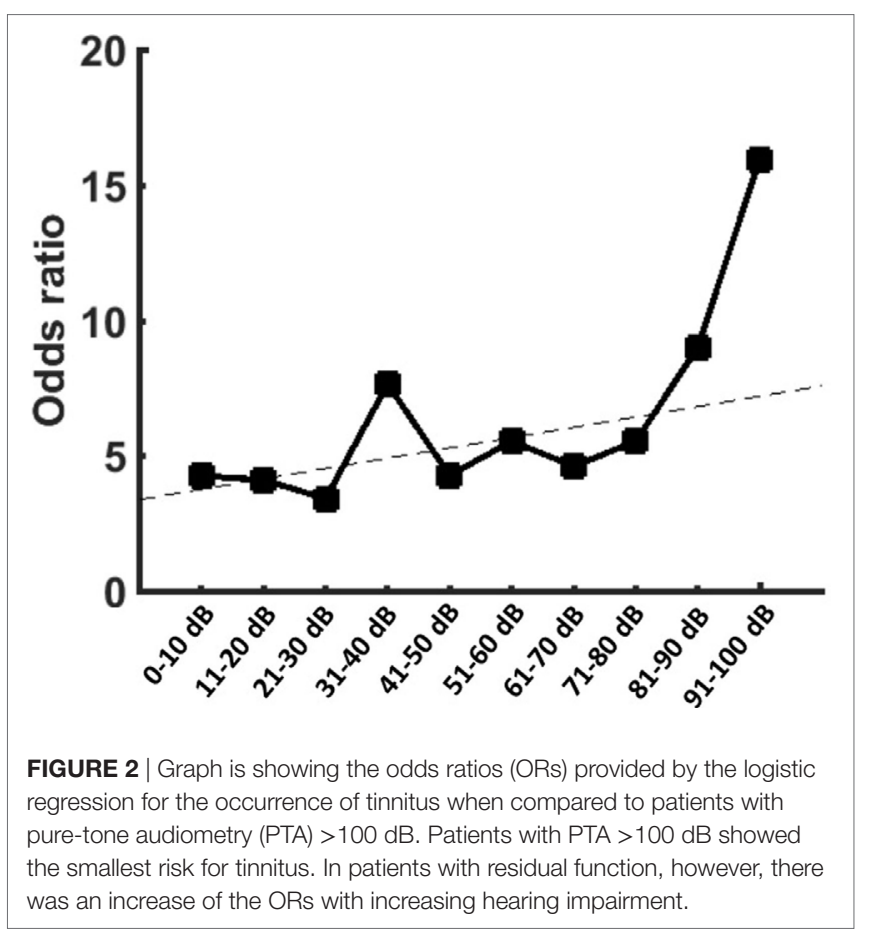




\section{Relationship between VS-Associated Tinnitus and Tumor Size}

So far, most studies evaluating the clinical predictors of VS-associated tinnitus have shown an inverse relationship between tumor size and tinnitus $(3,12,16-19)$. In line, our data show no predictive value of tumor size except the fact that patients with extreme large tumors (i.e., Hannover classification T4) are less likely to experience a tinnitus. Most of the available studies include VS patients treated mainly by the so-called translabyrinthine approach covering rather smaller VS $(5,12$, $16,20,24)$ In contrast, due to the retrosigmoidal approach used in our department, this study is covering a high proportion of extreme large tumors (i.e., Hannover classification T4) $(3,4,17$, $24,25)$. This fact might have contributed to our findings on the relation between tumor size and tinnitus. A detailed look at the statistics indicates that patients with small tumors (i.e., Hannover classification T1) have the largest risk of preoperative tinnitus in comparison to T2-T4 tumors, however, reaching statistical significance only in contrast to T4 tumors. To our opinion, the most likely explanation for this finding is that tinnitus might lead these patients to an early consultation with physicians, resulting in earlier detection of small tumors $(3,17)$. On the other, as T4 tumors are reaching the brain stem an interaction between tumor and the cochlear nucleus by compression cannot be excluded.

\section{Impact of Age, Tumor Side, and Gender on VS-Associated Tinnitus}

In this study, no correlation between patient's age or tumor side and VS-associated tinnitus was observed. In contrast, Kameda and colleagues found that patients suffering from VS-associated tinnitus are younger than patients without VS-associated tinnitus (17). Contradictory, Baguley and colleagues have shown a positive correlation between age and tinnitus severity, i.e., greater age being associated with greater tinnitus severity (12). Notably, in our analysis, male gender seems to be a positive predictor of VS-associated tinnitus. While there are some studies claiming a male preponderance in non-VS tinnitus $(26,27)$, most studies indicate female patients to be more affected (28-30). These gender differences could be related to different stress coping or anxiety sensitivity $(30,31)$. However, it remains unclear, how these findings refer to VS-associated tinnitus, when the tinnitus is superimposed by a tumor diagnosis and the need to surgical treatment. In such situation, men might exhaust their personal coping resources faster than women. Nevertheless, the relation between tinnitus and gender is still ambiguous. However, most studies demonstrate no association between tinnitus, gender, age, or VS tumor side $(4,5,18,20)$.

\section{Relationship between VS-Associated Tinnitus and Hearing Impairment}

Applying a multivariate analysis, this study is the first one to establish a significant, however, non-linear relationship between hearing impairment and VS-associated tinnitus. Our data show that higher preoperative hearing impairment is increasing the risk of developing a tinnitus while complete deafness, in turn, is reducing the risk. However, our data also show that $60 \%$ of the patients with good clinical hearing suffer from tinnitus. On the other hand, approximately $23 \%$ of the patients with complete hearing loss are suffering from preoperative tinnitus. For the first time, these findings indicate a non-linear relationship between hearing impairment and VS-associated tinnitus. To our opinion, this finding can be partially attributed to our retrosigmoidal approach, which resulted in a patient cohort with less preoperative hearing impairment $(3,4,17)$ than other studies applying mainly the translabyrinthine approach $(5,12$, $16,20,24)$. Our results are in good accordance with literature about non-VS tinnitus indicating hearing loss as main risk factor (7-10). Nevertheless, it is well known from non-VS tinnitus that clinical hearing impairment, as measured by the audiometry, is no necessary to develop tinnitus $(11,32,33)$. This finding could be explained by the fact that some forms of auditory deafferentation are not discovered by audiometry, e.g., patients with tinnitus who have normal hearing thresholds frequently are shown to have cochlear dead regions or outer hair cell damage compared with controls $(10,32)$. On the other hand, the sensitivity of the PTA might be insufficient to detect subtle (or hidden) hearing impairment $(11,33)$. In patients with nonVS tinnitus and normal hearing, Schaette and McAlpine have shown an association between tinnitus and a wave I amplitude reduction of the acoustic-evoked potentials, which is generated by primary auditory nerve fibers. This finding is supposed to be a physiological marker of reduced neural output from the cochlea. At the same time, however, the more centrally generated wave $\mathrm{V}$ presented a normal amplitude. These findings were interpreted as a renormalization of neuronal response magnitude to the reduced afferent input within the brainstem compensating the hearing loss (11). Our data show a significant increase of tinnitus occurrence in patients with non-serviceable hearing (GR3 and GR4) indicating that residual, possibly non-functional, activity of the acoustic nerve promotes the occurrence of tinnitus $(7-10)$. In line, there is evidence that cochlear nerve preservation with non-functional hearing seems to predict a new-onset tinnitus after surgical VS removal $(18,34)$. Finally, although hearing impairment could be the initial source of tinnitus, the subsequent cascade of maladaptive neuroplasticity in the central auditory system is likely perpetuate the tinnitus even without any residual input from the ear. This explains the occurrence of VS-associated tinnitus in patients with complete deafness and the fact that tinnitus is persisting even when afferent input from the ear is eradicated by cutting of the auditory nerve loss $(35,36)$. In contrast, re-installing functional hearing by cochlear implants has been shown to reduce tinnitus $(37,38)$.

\section{Limitations of the Study}

A major limitation of the study is the dichotomization of the patients' tinnitus complaints. Unfortunately, there are no systematic data on the tinnitus severity available loosing important information about the exact relation between tinnitus intensity and hearing impairment. Indeed, there are few studies using dedicated scores in VS-associated tinnitus. For example, Baguley and colleagues have shown a positive correlation between age and tinnitus severity. However, there was no significant association to hearing impairment (12). 


\section{CONCLUSION}

This study proves hearing impairment as the most important predictor of VS-associated tinnitus. This finding suggests similar pathophysiological mechanisms for VS-associated and non-VS tinnitus. A better understanding of the VS-associated tinnitus could pave the way for new therapeutic approaches (e.g., noninvasive neurostimulation).

\section{ETHICS STATEMENT}

This study was carried out in accordance with the recommendations of the ethics committee of the Eberhardt Karls University

\section{REFERENCES}

1. Moffat DA, Baguley DM, Beynon GJ, Da Cruz M. Clinical acumen and vestibular schwannoma. Am J Otol (1998) 19:82-7.

2. Myrseth E, Pedersen P-H, Møller P, Lund-Johansen M. Treatment of vestibular schwannomas. Why, when and how? Acta Neurochir (Wien) (2007) 149:647-60. doi:10.1007/s00701-007-1179-0

3. Matthies C, Samii M. Management of 1000 vestibular schwannomas (acoustic neuromas): clinical presentation. Neurosurgery (1997) 40:1-9. doi:10.1097/00006123-199701000-00001

4. Grauvogel J, Kaminsky J, Rosahl SK. The impact of tinnitus and vertigo on patient-perceived quality of life after cerebellopontine angle surgery. Neurosurgery (2010) 67:601-9. doi:10.1227/01.NEU.0000374725.19259.EA

5. Del Río L, Lassaletta L, Díaz-Anadón A, Alfonso C, Roda JM, Gavilán J. Tinnitus and quality of life following vestibular schwannoma surgery. B-ENT (2012) 8:167-71.

6. Lloyd SKW, Kasbekar AV, Baguley DM, Moffat DA. Audiovestibular factors influencing quality of life in patients with conservatively managed sporadic vestibular schwannoma. Otol Neurotol (2010) 31:968-76. doi:10.1097/ MAO.0b013e3181e8c7cb

7. Henry JA, Roberts LE, Caspary DM, Theodoroff SM, Salvi RJ. Underlying mechanisms of tinnitus: review and clinical implications. J Am Acad Audiol (2014) 25:5-22. doi:10.3766/jaaa.25.1.2

8. Baguley D, McFerran D, Hall D. Tinnitus. Lancet (2013) 382:1600-7. doi:10.1016/S0140-6736(13)60142-7

9. Shore SE, Roberts LE, Langguth B. Maladaptive plasticity in tinnitus - triggers, mechanisms and treatment. Nat Rev Neurol (2016) 12:150-60. doi:10.1038/ nrneurol.2016.12

10. Langguth B, Kreuzer PM, Kleinjung T, De Ridder D. Tinnitus: causes and clinical management. Lancet Neurol (2013) 12:920-30. doi:10.1016/ S1474-4422(13)70160-1

11. Schaette R, McAlpine D. Tinnitus with a normal audiogram: physiological evidence for hidden hearing loss and computational model. J Neurosci (2011) 31:13452-7. doi:10.1523/JNEUROSCI.2156-11.2011

12. Baguley DM, Humphriss RL, Axon PR, Moffat DA. The clinical characteristics of tinnitus in patients with vestibular schwannoma. Skull Base (2006) 16:49-58. doi:10.1055/s-2005-926216

13. Møller AR. Pathophysiology of tinnitus. Ann Otol Rhinol Laryngol (1984) 93:39-44. doi:10.1177/000348948409300110

14. O'Connor AF, France MW, Morrison AW. Perilymph total protein levels associated with cerebellopontine angle lesions. Am J Otol (1981) 2:193-5.

15. Sahley T, Nodar R, Musiek F. Efferent Auditory System: Structure and Function. San Diego: Singular Publishing Group, Inc. (1997).

16. Van Gompel JJ, Patel J, Danner C, Zhang AN, Samy YoussefAA, van Loveren HR, et al. Acoustic neuroma observation associated with an increase in symptomatic tinnitus: results of the 2007-2008 Acoustic Neuroma Association survey. J Neurosurg (2013) 119:864-8. doi:10.3171/2013.5.JNS122301

17. Kameda K, Shono T, Hashiguchi K, Yoshida F, Sasaki T. Effect of tumor removal on tinnitus in patients with vestibular schwannoma. J Neurosurg (2010) 112:152-7. doi:10.3171/2009.3.JNS081053
Tuebingen for retrospective studies of data collected as part of routine diagnosis and treatment.

\section{AUTHOR CONTRIBUTIONS}

GN and FE have contributed to the design of the work, the interpretation of the data, drafting and final approval of the manuscript and have agreed to be accountable for all aspects of the work. JS, ML, AO, AR, and GM have contributed to the acquisition and interpretation of the data, revising and final approval of the manuscript and have agreed to be accountable for all aspects of the work. MT has contributed to the interpretation of the data, revising and final approval of the manuscript and has agreed to be accountable for all aspects of the work.

18. Kohno M, Shinogami M, Yoneyama H, Nagata O, Sora S, Sato H. Prognosis of tinnitus after acoustic neuroma surgery - surgical management of postoperative tinnitus. World Neurosurg (2014) 81:357-67. doi:10.1016/j. wneu.2012.09.008

19. Myrseth E, Møller P, Wentzel-Larsen T, Goplen F, Lund-Johansen M. Untreated vestibular schwannomas: vertigo is a powerful predictor for health-related quality of life. Neurosurgery (2006) 59:67-76. doi:10.1227/01. NEU.0000219838.80931.6B

20. Alvarez L, Ugarte A, Goiburu M, Urreta Barallobre I, Altuna X. Change in tinnitus after acoustic neuroma removal using a translabyrinthine approach. A prospective study. Acta Otorrinolaringol Esp (2016) 67:315-23. doi:10.1016/j. otorri.2016.01.005

21. Samii M, Matthies C. Management of 1000 vestibular schwannomas (acoustic neuromas): hearing function in 1000 tumor resections. Neurosurgery (1997) 40:248-62. doi:10.1097/00006123-199702000-00005

22. Gardner G, Robertson JH. Hearing preservation in unilateral acoustic neuroma surgery. Ann Otol Rhinol Laryngol (1988) 97:55-66. doi:10.1177/000348948809700110

23. Gurgel RK, Jackler RK, Dobie RA, Popelka GR, Gurgel RK, Jackler RK, et al. Head and neck surgery reporting hearing outcome in clinical trials. Otolaryngol Neck Surg (2012) 147:803-7. doi:10.1177/0194599812458401

24. Chamoun R, MacDonald J, Shelton C, Couldwell WT. Surgical approaches for resection of vestibular schwannomas: translabyrinthine, retrosigmoid, and middle fossa approaches. Neurosurg Focus (2012) 33:E9. doi:10.3171/2012.6.FOCUS12190

25. Gharabaghi A, Samii A, Koerbel A, Rosahl SK, Tatagiba M, Samii M. Preservation of function in vestibular schwannoma surgery. Neurosurgery (2007) 60:ONS124-7. doi:10.1227/01.NEU.0000249245.10182.0D

26. George RN, Kemp S. A survey of New Zealanders with tinnitus. Br J Audiol (1991) 25:331-6. doi:10.3109/03005369109076606

27. Hiller W, Goebel G. Factors influencing tinnitus loudness and annoyance. Arch Otolaryngol Head Neck Surg (2006) 132:1323-30. doi:10.1001/ archotol.132.12.1323

28. Stouffer JL, Tyler RS. Characterization of tinnitus by tinnitus patients. J Speech Hear Disord (1990) 55:439-53. doi:10.1044/jshd.5503.439

29. Dineen R, Doyle J, Bench J. Audiological and psychological characteristics of a group of tinnitus sufferers, prior to tinnitus management training. Br J Audiol (1997) 31:27-38. doi:10.3109/03005364000000006

30. Seydel C, Haupt H, Olze H, Szczepek AJ, Mazurek B. Gender and chronic tinnitus. Ear Hear (2013) 34:661-72. doi:10.1097/AUD.0b013e31828149f2

31. Hesser H, Andersson G. The role of anxiety sensitivity and behavioral avoidance in tinnitus disability. Int J Audiol (2009) 48:295-9. doi:10.1080/ 14992020802635325

32. Weisz N, Hartmann T, Dohrmann K, Schlee W, Norena A. High-frequency tinnitus without hearing loss does not mean absence of deafferentation. Hear Res (2006) 222:108-14. doi:10.1016/j.heares.2006.09.003

33. Guest H, Munro KJ, Prendergast G, Howe S, Plack CJ. Tinnitus with normal audiogram: relation to noise exposure but no evidence for cochlear synaptopathy. Hear Res (2017) 344:265-74. doi:10.1016/j.heares.2016. 12.002 
34. Chovanec M, Zvěřina E, Profant O, Balogová Z, Kluh J, Syka J, et al. Does attempt at hearing preservation microsurgery of vestibular schwannoma affect postoperative tinnitus? Biomed Res Int (2015) 2015:783169. doi:10.1155/2015/783169

35. Jackson P. A comparison of the effects of eighth nerve section with lidocaine on tinnitus. J Laryngol Otol (1985) 99:663-6. doi:10.1017/ S0022215100097449

36. Bell JR, Anderson-Kim SJ, Low C, Leonetti JP. The persistence of tinnitus after acoustic neuroma surgery. Otolaryngol Head Neck Surg (2016) 155:317-23. doi:10.1177/0194599816642427

37. Blasco MA, Redleaf MI. Cochlear implantation in unilateral sudden deafness improves tinnitus and speech comprehension: meta-analysis and systematic review. Otol Neurotol (2014) 35:1426-32. doi:10.1097/ MAO.0000000000000431
38. Holder JT, O’Connell B, Hedley-Williams A, Wanna G. Cochlear implantation for single-sided deafness and tinnitus suppression. Am J Otolaryngol (2017) 38(2):226-9. doi:10.1016/j.amjoto.2017.01.020

Conflict of Interest Statement: There was no external funding of the study. None of the authors have potential conflicts of interest to be disclosed.

Copyright (c) 2017 Naros, Sandritter, Liebsch, Ofori, Rizk, Del Moro, Ebner and Tatagiba. This is an open-access article distributed under the terms of the Creative Commons Attribution License (CC BY). The use, distribution or reproduction in other forums is permitted, provided the original author(s) or licensor are credited and that the original publication in this journal is cited, in accordance with accepted academic practice. No use, distribution or reproduction is permitted which does not comply with these terms. 